\title{
DEMATERIALIZZAZIONE DELLA FISICA ${ }^{\#}$
}

\author{
GIACOMO MAURO D'ARIANO (*)
}

SunTO. - La Meccanica Quantistica ci ha insegnato una lezione che va ben oltre un insieme di nuove leggi fisiche: ci ha dotato di veri e propri "teoremi di epistemologia". Ora sappiamo che ci sono circostanze nelle quali l'osservazione del mondo non può essere concepita come mera lettura di una realtà locale che pre-esiste all'osservazione stessa. Sappiamo che ci sono circostanze nelle quali la conoscenza del tutto non implica la conoscenza delle parti (olismo). Sappiamo che esistono proprietà incompatibili che sono complementari, e che esistono proprietà del tutto che sono incompatibili con ogni proprietà di ognuna delle parti. La nozione di "oggetto" definito in termini delle sue "proprietà" entra quindi in contrasto con la sua connotazione mereologica secondo la quale gli oggetti si compongono formando nuovi oggetti. La stessa ontologia di particella come ente localizzabile è in contrasto con il teorema di Malament in teoria di campo. A questo aggiungiamo infine il fatto che le due grandi teorie fondamentali della fisica - la teoria quantistica dei campi e la relatività generale di Einstein - sono logicamente incompatibili. Per riconciliare la coerenza logica delle osservazioni con una loro spiegazione teorica occorre un cambio radicale di paradigma. La soluzione proposta è quella di abbandonare la visione del mondo come "meccanismo" e sostituirla con quella di "algoritmo". Ė questo il paradigma di "universo come grande computer" rimasto latente dai tempi di Richard Feynman, e che sta oggi riemergendo nella fisica, dimostrandosi di grande potenza teorica. Nella nuova visione algoritmica la "meccanica" diviene fenomenologia emergente. Non necessitando di primitive fisiche, il nuovo paradigma "informazionale" permette di fondare la fisica su solide basi assiomatiche. La teoria quantistica e la teoria di campo libera si derivano da assiomi informazionali. Gli assiomi della teoria quantistica hanno una connotazione epistemologica, e riguardano la possibilità di falsificare le proposizioni della teoria. Riconnettono logicamente olismo e riduzionismo, probabilismo e falsificabilità, sostituendo la nozione di "oggetto" con quelle di "sistema" e di "evento", e implicano logicamente i teoremi della teoria quantistica senza dover utilizzare spazi di Hilbert astratti. La teoria di campo libera

\# Testo integrale quasi verbatim della relazione presentata all'Istituto Lombardo Accademia di Scienze e Lettere nella giornata di studio Realtà senza Realismo del 7 giugno 2016.

(*) Dipartimento di Fisica dell'Università di Pavia, Italia.

E-mail: dariano@unipv.it 
(Weyl, Dirac e Maxwell) si deriva aggiungendo assiomi di minimizzazione della complessità algoritmica. La struttura informazionale separa nettamente "esperimento" e "teoria": l'esperimento è l'insieme degli eventi oggettivi, la teoria li connette mediante relazioni input-output, assegnandone le probabilità. La realtà oggettiva (esperimento) è fatta di "icone" con le quali interagiamo; la teoria è l'algoritmo soggiacente. Il paradigma che descrive il substrato della realtà è quello di puro software. "Software senza hardware": la dematerializzazione della fisica. "Realtà senza realismo" vuol dire puro "coerentismo" logico.

$* * *$

ABSTRACT. - Quantum Mechanics has taught us a lesson that goes well beyond a set of new physical laws. It has provided us with genuine "theorems of epistemology". We now know that there are situations when the observation of the world cannot be considered as the mere reading of a reality pre-existing the observation, whereas there are circumstances where the knowledge of the whole do not corresponds to the knowledge of the parts (holism). We know that there are incompatible properties that are complementary. We know that exist properties of the whole that are incompatible with any property of each part. The notion of "object" defined in terms of its "properties" is in contrast with its mereologic connotation according to which objects can be composed to form new objects. The particle ontology as localizable unity is in contrast with the Malament theorem in quantum field theory. To the above add the fact that the two more general fundamental theories in physics - the quantum theory of fields and the general relativity of Einstein - are logically incompatible. In order to reconcile the logical coherence of the observations with their theoretical explanation, we need a radical change of paradigm. The solution here proposed is to abandon the vision of the world as a "mechanism" and to substitute it with that of "algorithm". This is the paradigm of the "universe as a huge computer" that have been latent within the community since Richard Feynman, and nowadays is resurrecting in physics, showing its full theoretical power. In the new algorithmic vision the "mechanics" becomes an emergent phenomenology. Without the need of physical primitive, the new "informational" program allows us to found physics on solid axiomatic grounds. The quantum theory of abstract systems along with the free quantum field theory are derived from information-theoretic axioms. The axioms of quantum theory all have an epistemological connotation, and pertain the possibility of falsifying the propositions of the theory. They reconnect holism with reductionism, probabilism and falsifiability, substituting the notion of "object" with that of "system" and of "event", and logically implying the theorem of quantum theory without the need of using the abstract Hilbert-spaces toolkit. The free quantum field theory (Weyl, Dirac e Maxwell) is obtained by adding axioms of minimization of algorithmic complexity. The informational framework well separates the notions of "experiment" and "theory": the theory connects input with output, the experiment being identified with the collection of input, output, and objective intermediate events. The objective reality (experiment) is made of "icons" with which we interact: the theory is the underlying algorithm. The paradigm describes the tapestry of reality as pure "software". "Software without hardware": the dematerialization of physics. "Reality without realism" means pure logical coherentism. 


\section{ESSERE REALISTA SENZA ESSERE MATERIALISTA}

Per evitare un possibile fraintendimento del titolo della mia presentazione, devo premettere innanzitutto che mi definisco realista, intendendo con questo che assumo che esista una realtà indipendente da noi che la osserviamo e la descriviamo, e che le diverse descrizioni, al di là dei diversi linguaggi e dei diversi schemi teorici, convergono verso un omomorfismo - una corrispondenza di relazioni - con un substrato comune ai fenomeni osservati. Ciò ovviamente implica l'assunzione della traducibilità fra i diversi linguaggi, nonché l'ipotesi di una matematica e di una logica condivise.

Il punto è che essere realista nel senso suddetto non significa essere "materialista". Philip Clayton dice: A differenza della maggior parte delle posizioni filosofiche, che sono tenute ben distinte dalle teorie scientifiche, la visione del mondo materialista è sempre stata largamente accettata come implicazione diretta della scienza, quasi un sine-qua-non dell'attività scientifica [1]. Ebbene, essere realista non vuol dire credere in un mondo fatto di materia, particelle, energia, o dello spazio tempo incurvabile della relatività generale Einsteniana. Non vuol dire essere "meccanicista", o essere "determinista". L'errore che soggiace a questo tipo di realismo è quello della "realificazione" della teoria, ovvero l'identificazione degli oggetti della teoria - quali la particella o lo spazio tempo incurvabile - con elementi della realtà. La realificazione intrappola il paradigma scientifico nel meccanicismo deterministico, conducendo a forzature, quali le azioni istantanee a distanza della meccanica Bohmiana [2], oppure l'idea che la meccanica quantistica costituisca un nuovo tipo di logica, come pensavano Birkoff e von Neumann [3]. Personalmente credo che la logica della fisica e della scienza in genere debba essere la logica classica, e che questo sia necessario per poter soddisfare il criterio di demarcazione di Popper fra scienza e pseudoscienza [4]. Quello che invece è necessario è una riflessione seria sul metodo scientifico ed un cambio di paradigma. Ed è di questo di cui oggi desidero parlare.

\section{INFORMAZIONALISMO $^{1}$}

Oggi vi parlerò di un paradigma nuovo che sta emergendo in fisi-

1 Risultati preliminari della teoria basata sul paradigma informazionale furono 
$\mathrm{ca}$, in quella fisica teorica che studia il substrato più profondo della realtà, il suo stesso tessuto, ciò di cui sono fatte le particelle e lo spaziotempo. Un paradigma molto potente. Si tratta di quello che chiamiamo "informazionalismo", ovvero il paradigma "algoritmico" del mondo, da contrapporsi al paradigma corrente "meccanicistico" fatto di particelle che si urtano, interagiscono, o di stringhe o membrane che vibrano. Come già ha detto Kuhn, quando cambia il paradigma scientifico cambia la nostra visione del mondo [6]: creiamo una nuova ontologia. E il mio obiettivo oggi è quello di condurvi ad intuire una nuova visione del mondo, un mondo non fatto di particelle che si urtano e scambiano energia, bensì costituito da un immenso computer quantistico. Per dirla molto in breve, anticipando la sintesi della costruzione teorica che presenterò, il paradigma è quello di un universo fatto di "puro software". Non siamo noi che scriviamo il programma: noi cerchiamo di decodificarlo. E qual'è il programma? È semplicemente il programma più semplice che si possa scrivere.

È il paradigma informazionale preconizzato da John Archibald Wheeler e dallo stesso Richard Feynman [11], sintetizzato dallo slogan di Wheeler: "It from Bit" [12]. "software senza hardware": non c'è materia che supporta il software, ma la materia, gli atomi, le particelle, i fotoni, lo stesso spazio-tempo, e tutto quello che osserviamo non sono altro che icone tridimensionali risultanti da una soggiacente computazione quantistica, che avviene in un tessuto composto solamente da bit quantistici (per la precisione Fermionici).

In una visione realista meccanicista il paradigma "software senza hardware" sembra assurdo: come è possibile avere un software senza il supporto dell'hardware? Si può immaginare un supporto se lo si desidera, ma esso non ha una posizione nello spazio, non è fatto di "oggetti": l'ente elementare non ha altra caratteristica che quella di avere due stati discriminabili, 0 e 1, nonché le loro sovrapposizioni quantistiche. Il paradigma "software senza hardware" potrebbe sembrare all'antitesi del realismo Einsteiniano. Esso però identifica una nuova ontologia: l'ontologia quantistica digitale, di cui anche alcuni filosofi, come Luciano Floridi [13] e Terrel Bynum [14], stanno correntemente discutendo.

presentati in una precedente memoria presso lo stesso Istituto Lombardo [5]. Per articoli divulgativi dello stesso autore si veda $[7,8,9,10]$. 
Ma la grandezza della fisica teorica, ciò che la distingue dalla fantascienza, sono il rigore logico e la falsificabilità sperimentale, che devono esplicarsi essendo liberi da preconcetti, e capaci, anche di abbandonare un vecchio paradigma consolidato per esplorarne uno nuovo, se necessario. E il paradigma informazionale ha la grande potenzialità di rendere completa la coerenza logica della fisica teorica, risolvendone il problema dell'assiomatica posto da David Hilbert nel 1900 nel sesto dei suoi famosi ventitré problemi [15].

La motivazione fondamentale del mio discorso è quindi essenzialmente di natura metodologica: si tratta di ricostruire l'edificio della fisica su fondamenti solidi, per ritrovare una struttura logica coerente, che ne risolva le contraddizioni interne, fra le quali la più rilevante ed urgente resta l'incompatibilità fra teoria quantistica e relatività generale - le due più consolidate e generali teorie fisiche - incompatibilità a causa della quale non disponiamo ancora di una teoria microscopica della gravità. E l'informazionalismo, come vedremo, rappresenta un'opportunità unica - storicamente la prima - di risolvere il problema assiomatico della fisica, e potenzialmente di risolvere il conflitto fra la teoria quantistica e la relatività generale.

Devo premettere che, considerata la composizione eterogenea del pubblico, dovrò necessariamente esporre a due livelli paralleli, quello tecnico e quello divulgativo, intercalando affermazioni tecniche rigorose con illustrazioni intuitive divulgative dei concetti. Chiedo pertanto alla audience non tecnica di perdonare qualche termine specialistico, ma allo stesso tempo chiedo ai colleghi di tenere ben distinti i due livelli e di non fraintendere la divulgazione come argomentazione rigorosa. Ovviamente molti argomenti si basano su derivazioni teoriche che andrebbero ben al di là dei limiti di una relazione, e mi limiterò ad enunciarne i risultati di rilievo, facendo riferimento ai lavori pubblicati. Chiederei all'ascoltatore di concentrare l'attenzione sulla logica del discorso, più che sui contenuti specifici, e cercherò per questo di aiutarmi con dei diagrammi.

\section{INFORMAZIONALISMO QUANTISTICO}

La prima domanda è: "Perché quantistico il paradigma computazionale, e non classico?”

La Meccanica Quantistica ci ha insegnato una lezione che va ben 
oltre un insieme di nuove leggi fisiche: ci ha dotato di veri e propri "teoremi di epistemologia". Ora sappiamo che ci sono circostanze nelle quali l'osservazione del mondo non può essere concepita come mera lettura di una realtà locale pre-esistente l'osservazione, come ci dimostrano le violazioni delle disuguaglianze di Bell $[16,17,18]$. Ci sono circostanze nelle quali la conoscenza del tutto non implica la conoscenza delle parti, ovvero si ha olismo, come nel caso di due sistemi in uno stato massimamente entangled puro, ovvero noto a priori con assoluta precisione, laddove invece lo stato di ogni singolo sistema è completamente misto, ovvero completamente casuale, ignoto. Sappiamo che esistono proprietà incompatibili che sono complementari, come la posizione e il momento di una particella. Sappiamo che esistono proprietà del "tutto" che sono incompatibili con qualunque proprietà di ognuna delle "parti", come nel caso di una proprietà descritta tecnicamente da un proiettore entangled. La nozione di "oggetto" definito in termini delle sue "proprietà" entra quindi in contrasto con la sua connotazione "mereologica" secondo la quale gli oggetti si compongono formando nuovi oggetti. Infine, in teoria quantistica di campo la stessa ontologia di particella come ente localizzabile è in contrasto con il teorema di Malament [19]. Halvorson e Clifton [20] concludono che la teoria quantistica di campo non ammette un'ontologia di particella localizzabile, e strettamente parlando la particella localizzabile è una finzione. Quello che resta vero è parlare solo di proprietà di campi quantizzati e delle loro interazioni. Meinard Kuhlman dice [21]: I fisici normalmente descrivono l'universo come costituito di piccolissime particelle subatomiche, ma questo modo di vedere butta sotto il tappeto un fatto non molto conosciuto: l'interpretazione di particella della fisica quantistica, come anche quella di campo, abusano dei concetti di particella e di campo al punto che sempre più persone pensano che il mondo potrebbe essere fatto di qualcosa di completamente diverso.

Ottenere la meccanica quantistica da quella classica era il sogno rimasto irrealizzato di Einstein, che credeva di potere ridurre la teoria ad una meccanica statistica classica. Ma la violazione del bound di Bell ci insegna che le probabilità quantistiche non possono avere interpretazione epistemica (ovvero probabilità come mancanza di conoscenza di una realtà oggettiva ben definita), se non al prezzo di avere un realismo contestuale non locale, ovvero una realtà che si legge localmente ma dipende istantaneamente da ciò che viene fatto a distanza. La presenza di complementarità, ovvero l'esistenza di osservabili che non possono 
essere entrambe determinate, è una lezione ineludibile della meccanica quantistica, e il tentativo di Einstein di dimostrare l'incompletezza della teoria nel famoso articolo EPR [22] (dalle iniziali dei tre autori: Einstein, Podolski e Rosen) del 1935 si basava su un ragionamento controfattuale. La Meccanica Bohmiana [2] il cui fascino è innegabile (si veda la relazione di Nino Zanghì in questo volume), riduce meccanicamente le osservabili complementari della particella alla sola posizione, ma al caro prezzo di traiettorie frattali complicatissime e modificabili a distanza istantaneamente. Ma il prezzo più alto è che la richiesta di basare tutta la teoria sull'ontologia della particella entra in collisione con la possibilità di farne una teoria di campo relativistica. Dopo quasi novant'anni dalla prima formulazione di De Broglie e sessantacinque anni dal primo lavoro di Bohm [23] la teoria non riesce a descrivere processi di creazione e annichilazione di particelle; non si sanno descrivere particelle indistinguibili - Fermioni e Bosoni - e fra queste i fotoni, ovvero la teoria non sa descrivere il campo elettromagnetico quantistico. Altrettanto potremmo dire di una teoria dell'informazione classica digitale che emuli la teoria quantistica: si tratterebbe di una emulazione estremamente inefficiente: necessiterebbe di un numero fattoriale di connessioni input-output nonlocali fra tutti i bit classici coinvolti nella computazione. La lezione che abbiamo appreso dall'esperienza della nuova disciplina della Quantum Information, è che l'informatica quantistica è esponenzialmente più potente di quella classica, e la contiene come caso particolare.

\section{LA TEORIA QUANTISTICA È UNA TEORIA DELL'INFORMAZIONE}

La teoria quantistica generale dei sistemi astratti, ovvero la teoria privata delle sue connotazioni meccaniche - se volete la teoria dei bit quantistici o qubits - è una vera e propria teoria dell'informazione. Nel 2011 con Paolo Perinotti e Giulio Chiribella abbiamo derivato la teoria quantistica da principi di teoria dell'informazione [24]. ${ }^{2}$ Il lavoro è alla

2 Il lavoro ha ricevuto un Viewpoint dall'American Physical Society (che la società assegna ad uno ogni 2000 lavori pubblicati), è stato studiato da gruppi di ricerca con lavori e sezioni di conferenze dedicate, utilizzato da vari autori, ha ricevuto alcune centinaia di citazioni, è stato oggetto di capitoli di libri. 
base di un nuovo manuale in lingua inglese sulla teoria quantistica edito dalla Cambridge University Press [25].

\section{Il programma di assiomatizzazione}

Il lavoro [24] a livello metodologico rappresenta quello che dovrebbe essere il metodo di ricostruzione assiomatica della fisica, ovvero derivare la fisica da principi enunciati in assiomi puramente matematici, ma con interpretazione fisica. Assiomi che, in quanto matematici, non contengano primitive fisiche, come i concetti di "massa" e "forza", e i concetti di "orologio" e quello di "sbarra rigida" usati in teoria della relatività, nozioni per le quali lo stesso Einstein dichiarò il suo imbarazzo in svariate occasioni. Assiomi quindi puramente matematici, epurati delle primitive fisiche, ma con piena interpretazione fisica. L'interpretazione fisica è ovviamente essenziale, e deve propagarsi a tutti gli stadi della teoria, dagli assiomi fino alla formulazione dei teoremi, trasformandosi in un vero e proprio apparato concettuale per poter ragionare con la fisica senza dover dipendere solo dalle derivazioni teoriche. La necessità di un'interpretazione fisica sembra una ovvietà, ma l'assiomatica di von Neuman della teoria quantistica, che usiamo tutt'oggi, notoriamente non possiede una tale interpretazione, cosa che motivò il programma della quantum logic dello stesso von Neumann [3].

\section{La teoria quantistica è una teoria operazionale}

In sintesi, nella nostra assiomatizzazione la teoria quantistica è una teoria "operazionale" ${ }^{3}$ Nella teoria operazionale la nozione realistica di "oggetto" (che è una nozione teorica "realificata", contemporaneamente oggettiva/sperimentale e teorica, come il concetto di "particella"), viene sostituita dalle due nozioni di "evento" e di "sistema”, laddove l'evento è il dato sperimentale, l'accadimento oggettivo, mentre il sistema è una nozione teorica, e rappresenta una connessione input-output fra due eventi. In una teoria causale (ovvero che soddisfa l'assioma di causalità) il sistema rappresenta una connessione

3 Si veda anche la memoria [33] presentata allo stesso Istituto Lombardo da questo autore sulla derivazione della teoria quantistica da principi. 
causale. La connessione causale è quindi una nozione teorica, non oggettiva, affermazione che avrebbe trovato d'accordo David Hume.

Gli "eventi" sono i "fatti" del Tractatus Logico-Philosophicus di Ludwig Wittgenstein [26], che recita: "Il mondo è la totalità dei fatti, non delle cose." E la collezione di tutti i possibili eventi alternativi che possono accadere è quello che chiamiamo "test". La teoria operazionale è una teoria probabilistica dotata di regole di connettività fra gli eventi, ovvero connette gli eventi mediante connessioni inputoutput.

La nozione di "evento", e con essa quella di "test" - la collezione di tutti i possibili eventi alternativi - viene perciò "vestita" di "fili" di input sulla sinistra e di output sulla destra che ne rappresentano le connessioni con altri eventi (si veda Fig. 1).

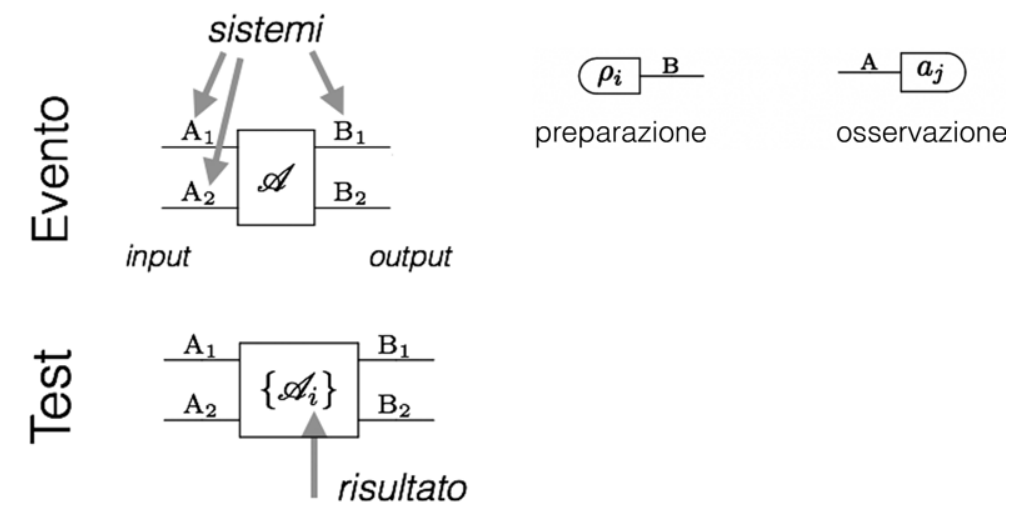

Fig. 1. Nella teoria operazionale la nozione di "oggetto" viene sostituita da quelle di "evento"

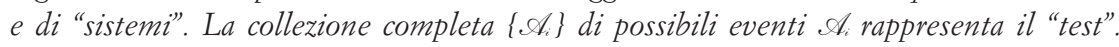
I sistemi introducono la connettività fra gli eventi. Eventi privi di input sono detti "preparazioni", eventi privi di output sono detti "osservazioni".

Come ogni buona teoria, la teoria operazionale contiene ben delineato l'ingrediente che descrive il dato sperimentale obiettivo, e per la teoria operazionale questo è il "risultato" (outcome) ovvero quale dei possibili eventi è avvenuto. L'evento è descritto in linguaggio sperimentale, e rappresenta quindi l'interfaccia fra il dato sperimentale obiettivo e la teoria. Esistono particolari eventi che non hanno input, detti "pre- 
parazioni", ed eventi che non hanno output, detti "osservazioni”, e si costruisce un circuito chiuso aciclico che, come ogni teoria, connette preparazioni con osservazioni (Fig. 2). Anche un Bohmiano non potrebbe fare a meno di assegnare la "preparazione", delimitando necessariamente la porzione di realtà descritta, altrimenti la sua teoria dovrebbe necessariamente descrivere il mondo intero ed essere in grado di specificarne o retrodirne le condizioni iniziali.

Ci si restringe quindi al solo aspetto probabilistico e si fa classe di equivalenza, definendo "trasformazione" della teoria la classe di equivalenza di tutti gli eventi che avvengono con la stessa probabilità congiunta se inseriti nello stesso circuito. La classe di equivalenza delle preparazioni è uno "stato" della teoria, mentre quella delle osservazioni è un "effetto".

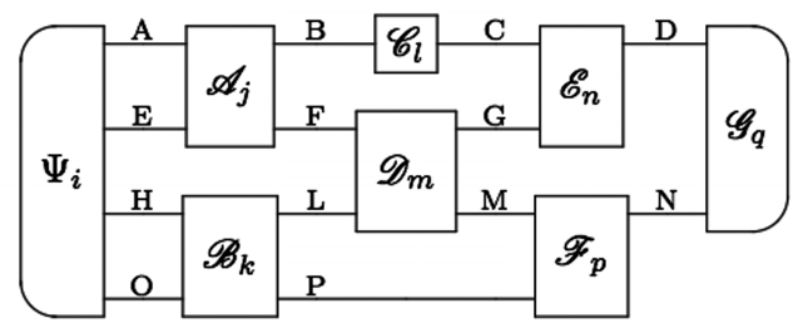

Fig. 2. Con gli eventi (o $i$ test) si costruiscono circuiti chiusi aciclici unidirezionali (da sinistra verso destra) ai quali si assegna la probabilità congiunta degli eventi. Pertanto, le probabilità congiunte sono parametrizzate da un circuito, ovvero denotate come $\mathrm{p}(\mathrm{i}, \mathrm{j}, \mathrm{k}, 1, \mathrm{~m}, \mathrm{n}, \mathrm{p}, \mathrm{q}$ lcircuit), e generalmente una probabilità marginale (ad esempio $\mathrm{p}(\mathrm{i}, \mathrm{j}, \mathrm{k} \mid$ circuit $)=\Sigma_{l, m, n, q} \mathrm{p}(\mathrm{i}, \mathrm{j}, \mathrm{k}, \mathrm{l}, \mathrm{m}, \mathrm{n}, \mathrm{p}, \mathrm{q}$ lcircuit $\left.)\right)$ dipende ancora dal circuito complessivo, ovvero anche dalla scelta dei test $\left\{\mathscr{G}_{l}\right\},\left\{\mathscr{D}_{m}\right\},\left\{\mathscr{E}_{n}\right\},\left\{\mathscr{F}_{p}\right\} e\left\{\mathscr{G}_{q}\right\}$, nonché dalle loro connessioni.

Quindi, in definitiva si ha un circuito chiuso direzionato da sinistra a destra che inizia con stati e si chiude con effetti. La costruzione del circuito si ottiene per composizione in serie e in parallelo di trasformazioni e di sistemi. In linguaggio matematico la teoria operazionale corrisponde a una categoria strettamente monoidale simmetrica. L'interpretazione fisica è quella delle operazioni fatte in laboratorio, o più generalmente quella di un "processo": è il run di un programma computazionale, un processing di informazione. 


\section{La teoria quantistica è un'estensione della logica}

Ricapitolando, la teoria operazionale associa ad ogni distribuzione di probabilità congiunta di eventi un circuito chiuso che ne rappresenta le connessioni teoriche. La teoria quantistica quindi, in quanto teoria operazionale, è un'estensione della teoria delle probabilità, la quale a sua volta è un estensione della logica, come dimostrano Edwin Jaynes [27] e Richard T. Cox [28]. Non si tratta quindi di modificare la logica, come intendeva von Neumann [3], bensì di estenderla, come in un qualsiasi processo di inferenza. E l'estensione è una teoria operazionale, ovvero una teoria dell'informazione.

Il paradigma è quindi non quello del fisico teorico "meccanico" che cerca di determinare il meccanismo di funzionamento del mondo, bensì quello di un backer informatico che decodifica il programma del software di cui è fatto il mondo. Quello che noi vediamo, gli eventi oggettivi, non sono altro che "icone" con le quali interagiamo in una sorta di enorme schermo multidimensionale, del quale noi stessi facciamo parte, con immagini 3D, suoni, forze, gravità, chimica, odori. La teoria è l'algoritmo soggiacente che noi vogliamo decodificare, che corre su qubits, e l'hardware che esegue l'algoritmo è del tutto irrilevante.

\section{PRINCIPI PER LA TEORIA QUANTISTICA ${ }^{4}$}

Ma quali sono le regole di questo software? La teoria quantistica è il sistema operativo. Nella teoria operazionale si definiscono gli assiomi della teoria quantistica:

1. Causalità,

2. Discriminabilità perfetta,

3. Discriminabilità locale,

4. Atomicità della composizione,

5. Compressione ideale,

6. Purificazione.

Analizziamo succintamente solo l'assioma numero uno: la causalità. Qualunque circuito chiuso si può vedere come una preparazione seguita da un'osservazione (si veda Fig. 3). L'ingrediente non banale da

4 Per la teoria di questa sezione si veda il testo [25]. 
rimarcare è che in una teoria operazionale la dipendenza parametrica da un circuito della probabilità congiunta implica che se si marginalizza su un test del circuito, la distribuzione di probabilità marginale generalmente dipende ancora dal test marginalizzato. Quello che afferma il postulato di causalità è che la probabilità marginale della preparazione è indipendente dalla scelta del test di osservazione.

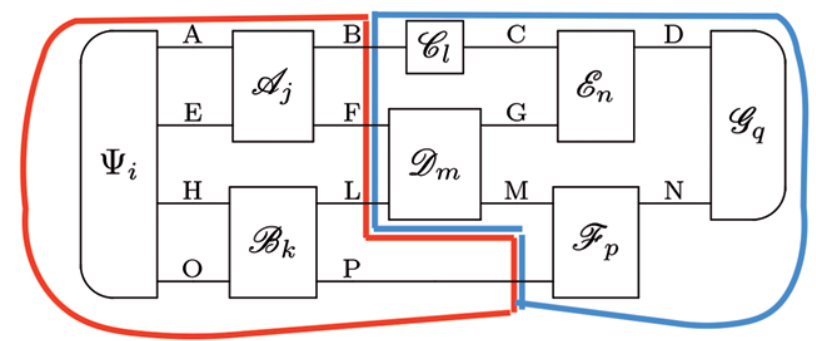

Fig. 3. Ogni circuito chiuso può essere suddiviso in una preparazione (porzione di circuito a sinistra), ed una osservazione (porzione di circuito a destra). Il postulato di causalità stipula che la probabilità marginale di preparazione $\mathrm{p}(\mathrm{i}, \mathrm{j}, \mathrm{k} \mid$ circuit $)={ }_{\mathrm{l}, \mathrm{m}, \mathrm{n}, \mathrm{p}, \mathrm{q}}$ $\mathrm{p}(\mathrm{i}, \mathrm{j}, \mathrm{k}, \mathrm{l}, \mathrm{m}, \mathrm{n}, \mathrm{p}, \mathrm{q}$ lcircuit) non dipenda dalla scelta dell'osservazione, ovvero non dipenda dalla porzione di circuito a destra connessa all'output della preparazione.

Il postulato di causalità è molto potente, se si pensa che nel 1982 Nick Herbert proponeva sulla rivista Foundation of Physics [29] (nonchè brevettava!) un metodo di comunicazione istantanea a distanza basato sulla condivisione di uno stato entangled, ottenendo cospicui finanziamenti dal Pentagono, come riportato sul libro di Davi Kaiser How the Hippies Saved Physics [30]. La prima dimostrazione generale dell'impossibilità di comunicare senza interazione risale al 1980 [31]. Il fraintendimento dell'entanglement con la possibilità di comunicare istantaneamente era molto comune, e in parte indotto dall'espressione spiritosa di Einstein Spooky action at distance (azione "fantasmatica" a distanza). E la non ovvietà dell'impossibilità di comunicare superluminalmente in presenza della nonlocalità quantistica era il cavallo di battaglia di Abner Shimony che usava l'espressione: Peaceful coexistence between Quantum Mechanics and Special Relativity.

L'impossibilità di comunicare senza interazione condividendo uno stato entangled è semplicemente una conseguenza immediata della causalità. Il circuito che descrive la condivisione di uno stato entangled 
fra Alice e Bob che eseguono osservazioni locali può essere diviso in una preparazione che include lo stato entangled e anche la misura di Alice, e un'osservazione che contiene solo la misurazione di Bob (si veda Fig. 4). L'indipendenza della probabilità di preparazione di Alice dalla scelta della osservazione di Bob sancisce l'impossibilità di comunicare superluminalmente da Bob verso Alice (e similmente scambiando i ruoli di Alice e Bob).
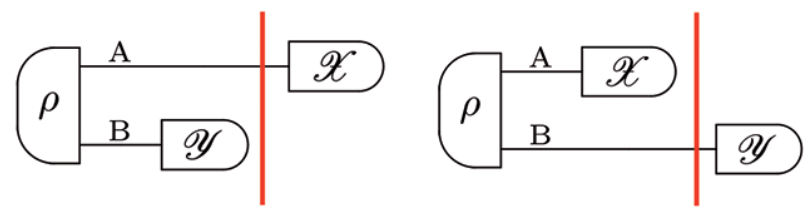

Fig. 4. Dimostrazione grafica dell' impossibilità di comunicare istantaneamente a distanza mediante la condivisione di uno stato entangled. Nel diagramma a sinistra, la preparazione (corrispondente allo stato entangled) insieme all'osservazione YY di Bob costituiscono una nuova preparazione, la cui probabilità congiunta è quindi indipendente (per la causalità), dalla osservazione $\mathscr{X}$ di Alice. Pertanto quel che Bob rivela è indipendente dalla operazione di Alice, e Alice non comunica nulla a Bob. Similmente nel diagramma a destra, con i ruoli di Alice e Bob scambiati.

\section{Rilevanza epistemologica dei postulati per la falsificabilità della teoria}

Tutti e sei i postulati hanno una rilevanza epistemologica, e riguardano la falsificabilità delle proposizioni della teoria. La causalità stessa è necessaria per garantire un cono spazio-temporale di schermatura dell'esperimento, assicurando che la falsificazione non sia dovuta ad un'azione a distanza o dal futuro in una teoria dotata di località (della località parleremo fra breve). Si noti che questa nozione di causalità corrisponde anche all'uso standard della causalità nei processi di inferenza $\mathrm{e}$ di modellizazione (si veda il libro sulla causalità di Judea Pearl [32]), e si può dimostrare che è equivalente alla causalità Einsteniana. La discriminabilità perfetta garantisce la falsificazione delle proposizioni della teoria. La discriminabilità locale garantisce che per proposizioni nonlocali la falsificazione sia ottenibile con osservazioni locali, riconciliando l'olismo della teoria con l'approccio riduzionista. L'atomicità della composizione garantisce che la composizione di trasformazioni non produca perdita di informazione riguardo la trasformazione risultante. La comprimibilità ideale garantisce che ogni sorgente di informazione 
ridondante possa essere compressa in modo reversibile ad una sorgente non ridondante.

Mentre i primi cinque assiomi sono comuni alla teoria classica, l'assioma di purificazione è quello che discrimina la teoria quantistica. Concettualmente è l'espressione della conservazione dell'informazione. Dal principio consegue che l'irreversibilità è sempre riconducibile ad una mancanza di controllo su un "environment", ovvero che ogni processo irreversibile può essere ottenuto da un processo reversibile di interazione con un environment.

\section{FISICA SENZA FISICA: DA DOVE VIENE LA MECCANICA?}

Fino ad ora abbiamo visto solo la teoria quantistica dei sistemi astratti. Sorgono spontanee le domande: "da dove provengono concettualmente le "particelle"? Come si fa ad ottenere la meccanica senza primitive fisiche negli assiomi? Fisica senza fisica? Fisica come pura interpretazione della matematica?

Abbiamo derivato i sistemi quantistici e le loro interazioni. Consideriamo quindi semplicemente un insieme contabile di sistemi quantistici in interazione, ed aggiungiamo alcuni principi generali che regolano queste interazioni. I principi sono:

1. Unitarietà,

2. Località,

3. Omogeneità,

4. Isotropia.

Tutti gli assiomi corrispondono al principio generale di semplicità dell'algoritmo, di minimizzazione della sua complessità. L'evoluzione è descritta da un unico operatore unitario che corrisponde ad una singola step di evoluzione discreta. La rete di interazioni fra i sistemi si traduce nella costruzione di un grafo colorato e direzionato di cui i sistemi sono i nodi e i links colorati descrivono le diverse interazioni.

L'assioma di unitarietà è una conseguenza immediata del fatto che i sistemi sono quantistici, perchè qualunque evoluzione quantistica si può ottenere mediante un'evoluzione unitaria, la quale è quindi la più semplice. L'assioma di località stabilisce che c'è un numero massimo di sistemi

5 Per questa sezione si veda la teoria nell'articolo [35]. 
che interagiscono con ogni sistema, e quindi l'informazione non può fluire da un sistema all'altro se non percorrendo il grafo. L'assioma di omogeneità stabilisce nel modo più generale che il circuito delle connessioni fra i vari sistemi è lo stesso visto da ogni sistema. Matematicamente, esiste un gruppo di permutazioni che può scambiare qualunque coppia di sistemi e che commuta con la dinamica, ovvero è una simmetria. Ne consegue che il numero di sistemi che interagiscono con ogni sistema è uguale per tutti i sistemi, il ventaglio dei link colorati attorno ad ogni sistema è lo stesso per tutti i sistemi, ed infine che tutte le loop colorate che contengono un sistema sono le stesse per ogni sistema. L'assioma di isotropia corrisponde a richiedere che esiste un gruppo di permutazione dei colori che corrisponde ad un automorfismo del grafo - ad esempio a ruotarlo ed un corrispondente gruppo di trasformazioni unitarie dei sistemi che assieme lasciano invariata la dinamica. Questo corrisponde alla nozione di "spin": se si ruota il grafo e allo stesso tempo si "ruota" il sistema, la dinamica risultante resta invariante.

Dai quattro assiomi discende che il grafo è il grafo di Cayley di un gruppo $G$ finitamente presentato, dove i siti rappresentano elementi del gruppo $G$, ed il colore delle frecce è in corrispondenza con un generatore del gruppo, e la freccia rappresenta l'operazione del generatore sull'elemento del gruppo da cui parte la freccia con risultato l'elemento alla punta della freccia (si veda Fig. 5). La dinamica è quindi descritta da un automa cellulare quantistico che ha come grafo il grafo di Cayley di un gruppo $G$ finitamente presentato, e nel caso lineare è un quantum walk sul Cayley graph.

\section{Una nuova matematica: la geometric group theory}

La suddetta costruzione matematica, che segue direttamente dagli assiomi, collega la nuova teoria di campo ad una branca della matematica pura contemporanea, la geometric group theory [36, 37].

Devo qui rimarcare che Einstein stesso aveva considerato che lo spazio tempo non fosse continuo, bensì sussistesse una possibile costruzione discreta, ma lamentava l'assenza di una matematica adeguata. ${ }^{6}$

6 Einstein scriveva: But you have correctly grasped the drawback that the continum brings. If the molecular view of matter is the correct (appropriate) one, i.e., if a part of the universe is to be represented by a finite number of moving points, then the continum 


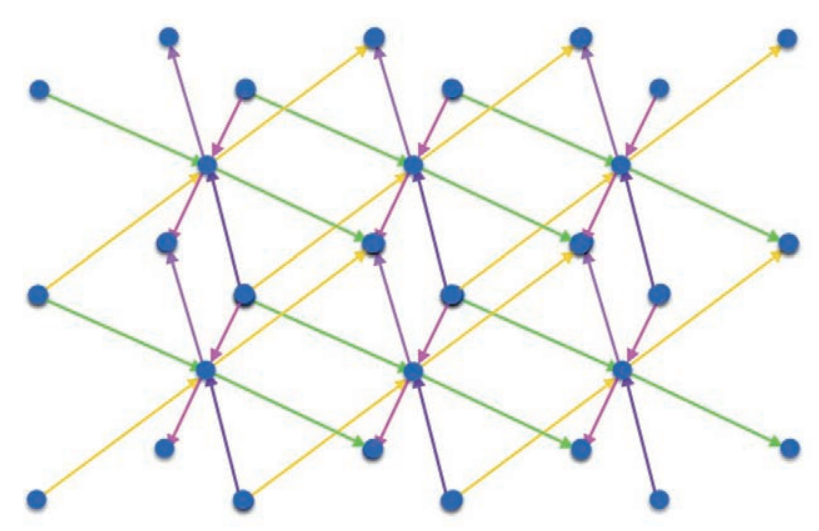

Fig. 5. Grafo di Cayley del gruppo Abeliano $\mathbb{Z}^{3}$, corrispondente ad una presentazione con quattro generatori del gruppo. Il reticolo è il BCC (body centered cubic).

Questa matematica oggi esiste, ed è appunto la geometric group theory. Nata all'inizio degli anni '90 da un'idea di Mikhail Gromov, consiste nello studiare le proprietà di un gruppo finitamente presentato in termini delle proprietà dello spazio metrico nella quale i suoi grafi di Cayley si embeddano "quasi-isometricamente", conducendo ad una geometrizzazione del problema gruppale. Ad esempio, restringendoci a gruppi infiniti che rappresentano il caso di interesse, teoremi dovuti a Gromov e altri matematici dimostrano che lo spazio metrico in cui si embedda il gruppo è Euclideo se e solo se il gruppo $G$ è virtualmente Abeliano, oppure se $G$ non è virtualmente nihilpotente lo spazio è iperbolico. Nel nostro caso lo spazio metrico nella teoria rappresenta lo spazio che emerge dalle interazioni fra i qubits. La restrizione di embeddabilità a spazi Euclidei corrisponde a ritrovare la fisica nello spazio Euclideo, e restringe il gruppo ad essere virtualmente Abeliano.

of the present theory contains too great a manifold of possibilities. I also believe that this too great is responsible for the fact that our present means of description miscarry with the quantum theory. The problem seems to me how one can formulate statements about a discontinuum without calling upon a continuum (space-time) as an aid; the latter should be banned from the theory as a supplementary construction not justified by the essence of the problem, which corresponds to nothing real?. But we still lack the mathematical structure unfortunately. How much have I already plagued myself in this way! [38]. 
L'automa cellulare quantistico più semplice: il Fermione di Weyl

Per dirla in breve, si dimostra che se si considera l'automa cellulare più semplice che deriva dalla teoria embeddabile nello spazio Euclideo $\mathbb{R}^{3}$, esso corrisponde al gruppo Abeliano $\mathbb{Z}^{3}$ delle traslazioni. Si dimostra che ci sono solo due automi con un campo Fermionico con spin $1 / 2$, che nel limite relativistico di piccoli vettori d'onda danno esattamente le due equazioni di campo di Weyl per particella left-handed e right-handed senza massa. ${ }^{7}$ Come vedremo la scala di piccolezza dell'automa è la scala di Planck, che è una scala piccolissima, per la quale in proporzione se prendessimo una lunghezza di Planck pari ad un centimetro un elettrone sarebbe grande come una galassia. Viceversa, l'energia di Planck è enorme, pari a $10^{28} \mathrm{eV}=.5 \mathrm{MWh}$. In unità Planckiane i raggi cosmici ultra energetici osservati alcuni anni orsono (che sono enormemente più energetici di quelli ottenibili al LHC di Ginevra e non sono spiegabili all'interno del cosiddetto modello Standard delle particelle), hanno vettor d'onda di un centomilionesimo: quindi tutto quello che abbiamo osservato nella storia della fisica rientra ampiamente nel limite relativistico.

Componendo due automi di Weyl in somma diretta e prodotto tensore in modo da soddisfare ancora tutti i principi, otteniamo nuovi automi. Ebbene questi, nel limite relativistico, danno l'equazione di Dirac per particelle con massa nonché le equazioni di Maxwell per i fotoni. Otteniamo quindi la teoria quantistica relativistica di campo libera.

\section{PREVISIONI DELLA TEORIA}

La precisione con la quale la dinamica discreta riproduce la teoria di campo libera è purtroppo ottima: dico "purtroppo" perché gli effetti dovuti alla discretezza sono troppo piccoli per essere osservati. Questi si possono riassumere nel fatto che lo spazio vuoto si comporta un po' come un solido cristallino, e l'effetto più visibile è la dispersione, ovvero la dipendenza della velocità della luce dalla lunghezza d'onda [39]. Benchè l'effetto sia piccolissimo si accumula su grandissime distanze,

7 Il fatto che il gruppo sia Abeliano tecnicamente ci permette di introdurre il concetto di vettore d'onda. 
ed è potenzialmente visibile nella rivelazione di raggi gamma ad altissime energie provenienti da quasar ai limiti dell'universo visibile. In tal caso vedremmo pervenire i pacchetti a lunghezze d'onda molto diverse con un ritardo temporale di alcuni secondi fra loro. Eventi gamma di questo tipo sono visibili potenzialmente dal Fermi Gamma-ray Space Telescope. Un altro effetto è quello che i fotoni risultano in realtà composti da una coppia di Fermioni entangled (come in una vecchia teoria di de Broglie [40]), ma questo effetto non è visibile nemmeno con la radiazione più potente che abbiamo a disposizione, quella del Vulcan laser. ${ }^{8}$ Infine, un effetto combinato della discretezza e dell'unitarietà è il fatto che la massa dell'automa di Dirac è limitata superiormente. Il fatto che la massa di una particella sia limitata superiormente è anche previsto dalla teoria dalla relatività generale in combinazione con la teoria quantistica di campo, che prevedono euristicamente che se la massa è più grande della massa di Planck la particella diventerebbe un buco nero'. Anche nel caso dell'automa l'informazione smette di propagarsi, per cui euristicamente si deduce che il valore della massa massima sia dell'ordine di grandezza della massa di Planck.

\section{La teoria contiene le unità di misura}

È davvero notevole che la teoria fondata su assiomi puramente matematici - e quindi necessariamente con tutte le variabili adimensionali - contenga al suo interno le misure campione attraverso la discretezza stessa della teoria ed il valore massimo della massa. Questo non sarebbe possibile per una teoria nel continuo. Il valore della massa massima $m_{*}$ potrebbe essere determinato sperimentalmente da un ritardo dei pacchetti da rivelazione di raggi gamma ad altissime energie provenienti da quasar ai limiti dell'universo, visibili come già detto dalla missione Fermi, mentre, nota la massa massima $m_{*}$, il valore dell'unità di tempo $t_{*}$ e dell'unità di spazio $a_{*}$ sarebbero determinati dalla velocità della luce a bassa frequenza $\mathrm{c}=a_{*} / t_{*}$ e dall'interessante relazione $\hbar=m_{*} a_{*} t_{*}$ che li lega alla costante di Planck $\hbar$, e che attribuisce alla

8 Il Vulcan laser ha una potenza di 1 PW, pari alla potenza di 50 centrali elettriche delle più potenti.

9 In tal caso, infatti, la lunghezza d'onda Compton della particella, corrispondente al raggio di localizzazione, diventerebbe uguale al raggio di Schwartzild. 
costante un'interpretazione puramente meccanica, come deve essere, essendo una costante con dimensioni meccaniche.

\section{Fisica da informazione e relatività}

È anche rimarchevole che la teoria relativistica di campo sia stata ottenuta senza assumere nè meccanica, nè cinematica, ma soprattutto senza assumere la relatività ristretta, e nemmeno il principio di Galileo. ${ }^{10} \mathrm{Ma}$ cos'è un sistema di riferimento in un universo discreto senza spazio-tempo? La nozione di sistema di riferimento non è altro che la rappresentazione della dinamica, e pertanto i sistemi di riferimento inerziali sono le rappresentazioni che lasciano la dinamica invariante. I cambi di rappresentazione formano un gruppo che per massa zero è una versione nonlineare del gruppo di Lorentz che nel limite relativistico di piccoli vettori d'onda recupera perfettamente il gruppo delle trasformazioni di Lorentz usuali della relatività. Invece, alle energie elevatissime della scala di Planck si hanno distorsioni che evidenziano la granularità quantistica dello spazio-tempo.

Per massa diversa da zero, invece, anche la massa a riposo della particella viene coinvolta nelle trasformazioni, e il gruppo diventa quello di De Sitter, che coincidentalmente nella teoria della relatività generale è una soluzione altamente simmetrica di vuoto che richiede costante cosmologica, e supporta l'idea di una relatività de Sitter-invariante anzichè invariante sotto il gruppo di Poincaré. Questa coincidenza, insieme all'euristica del mini buco nero già citata, ha un sapore promettente di relatività generale. Come anche promettente è il fatto che la massa $m \in[-1,1]$ in realtà ha la topologia di un cerchio, in quanto ai valori estremi +1 e -1 le dinamiche coincidono. ${ }^{11}$ La topologia del cerchio ci dice che la sua variabile coniugata, che secondo la meccanica Hamiltoniana è il tempo proprio, è discreta: ciò rappresenta una non ovvia consistenza interna della teoria.

Il cerchio si chiude: ritroviamo la relatività di Einstein, ritroviamo

10 Si rammenta che il principio di Galileo dell'invarianza per riferimenti inerziali delle leggi fisiche, coincide con quello di Einstein della relatività ristretta, a patto di includere l'elettromagnetismo.

11 Si noti che nella teoria interagente la massa negativa corrisponderà a carica elettrica opposta. 
il concetto di particella come simmetria, troviamo gli standard di lunghezza, tempo e massa, e sembra di vedere all'orizzonte una luce che promette uno spiraglio sulla gravità. Lo schema logico complessivo della derivazione da principi informazionali della teoria di campo quantistica libera è riportato in Fig. 6.

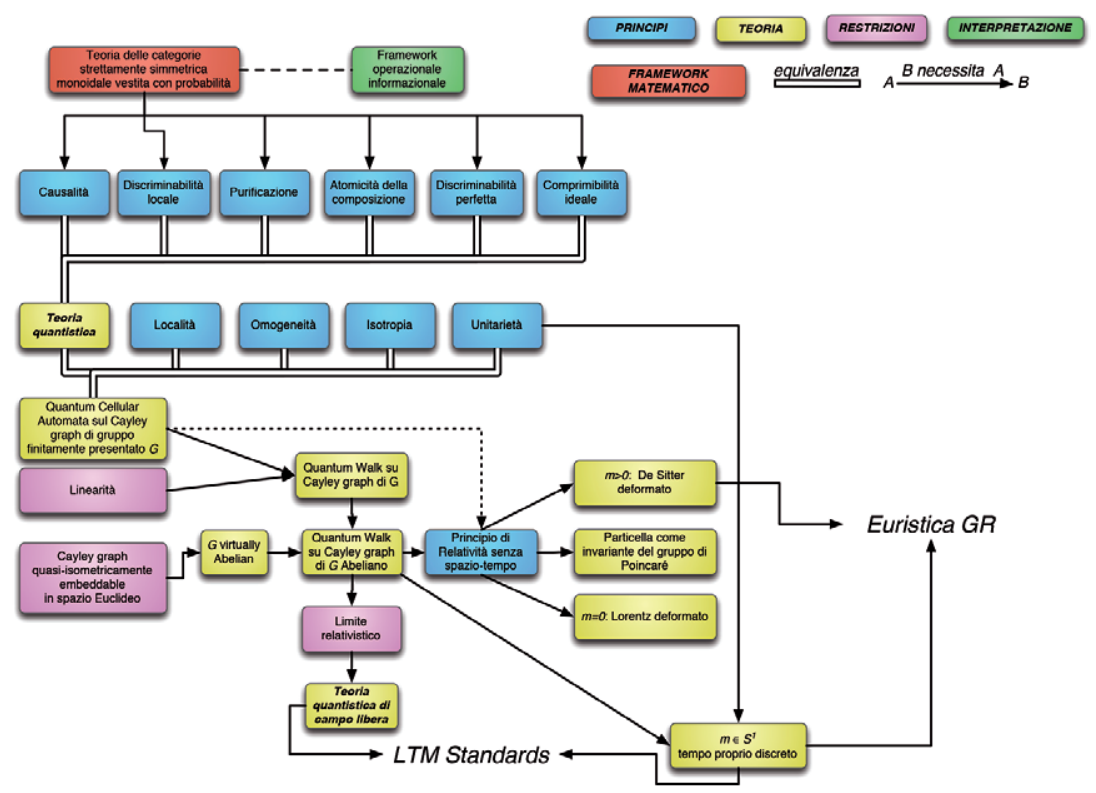

Fig. 6. Schema logico generale della derivazione da principi informazionali della teoria di campo quantistica libera.

Il ruolo dell'informazione quantistica nella teoria della gravità

È ormai assodato dalla letteratura recente sui buchi neri che l'informazione quantistica deve giocare un ruolo cruciale in quello che sarà la teoria microscopica della gravità, la cosiddetta quantum gravity, come avviene ad esempio per il famoso bound di Bekenstein che connette l'entropia di un buco nero all'area del suo orizzonte degli eventi, e che è connesso al principio olografico, ovvero lo spazio-tempo dell'universo è descrivibile da una superficie poliedrica con facce di area Planckiana, ognuna corrispondente a un qubit d'informazione. 


\section{PRINCIPI NON FALSIFICABILI?}

Concludo con alcune considerazioni generali sui principi dai quali è stata dedotta assiomaticamente la teoria. Come abbiamo visto, $\mathrm{i}$ principi della teoria quantistica riguardano tutti la falsificabilità delle proposizioni della teoria: in quanto tali, si potrebbe concludere che per definizione non sono falsificabili. Resta comunque il fatto che possano esistere variazioni dei principi, quali ad esempio un indebolimento del principio della Discriminabilità locale che conduce alla teoria quantistica Fermionica, oppure la sostituzione del principio della Purificazione con quello della Discriminabilità perfetta degli stati puri, che darebbe la teoria classica, e che quindi da una lato eliminerebbe la complementarità, ma dall'altro non permetterebbe di falsificare valori non deterministici ( 0 o 1$)$ delle probabilità, cosa che è garantita invece dal principio di Purificazione.

Per quanto riguarda i principi di omogeneità e di isotropia - ma anche lo stesso principio di relatività, che non è stato utilizzato come assioma, bensì derivato dagli assiomi - si tratta in tutti i casi di principi di natura convenzionale. Spiego cosa vuol dire con alcuni esempi storici.

Hans Reichenbach argomentava circa la convenzionalità dell'uguaglianza delle velocità di andata e di ritorno della luce, notando che per sincronizzare orologi distanti mediante segnali luminosi occorre conoscere la velocità della luce su un percorso aperto, ma d'altra parte per determinare la velocità della luce su un percorso aperto occorre possedere due orologi sincronizzati distanti. Si ha quindi un circolo vizioso. Possiamo solo conoscere la velocità della luce su un percorso chiuso utilizzando un unico orologio. L'uguaglianza delle velocità di andata e ritorno della luce è quindi convenzionale. Wesley Salmon conclude allora che la simultaneità non solo è relativa al sistema di riferimento, bensì all'interno dello stesso sistema di riferimento essa è convenzionale, come conseguenza della convenzionalità dell'uguaglianza delle velocità di andata e ritorno.

Micheal Friedman rigetta la convenzionalità della simultaneità, in quanto la struttura di spazio-tempo Minkowskiano è intimamente connessa alla simultaneità alla Einstein, e una convenzione diversa distruggerebbe l'intera costruzione. In definitiva assume come motivazione della nozione Einsteiniana di simultaneità un argomento di semplicità della teoria.

Adolf Grübaum argomenta che lunghezze in luoghi diversi non 
sono confrontabili, e altrettanto vale per gli intervalli di tempo, e pertanto le nozioni di congruenza sia spaziali che temporali sono convenzionali. Similmente Reichenbach decreta che l'uniformità dell'orologio è motivata solo dalla semplicità descrittiva, e che l'orologio più preciso è quello che semplifica la coerenza logica interna dell'intera descrizione teorica.

In definitiva, omogeneità e isotropia di spazio e tempo sono convenzionali, e la motivazione generale sottostante a tutte queste convenzioni è solo la semplicità della teoria. Anche nel nostro caso, i principi di omogeneità ed isotropia sono dettati dalla minimizzazione della complessità algoritmica dell'algoritmo quantistico che rappresenta la legge fisica.

\section{CONCLUSIONI}

La lezione che abbiamo imparato è che molta fisica, forse tutta la fisica, discende da principi di epistemologia, che riguardano la falsificabilità stessa della teoria, e il nostro stesso modo di fare scienza. Descrivendo la natura, riscopriamo un po' di noi stessi. E riscopriamo la saggezza Socratica del $\gamma v \tilde{\omega} \theta \mathrm{\imath} \sigma \varepsilon \alpha v \tau o ̀ v$, ovvero: "conosci te stesso".

\section{BIBLIOGRAFIA}

[1] P. Clayton, Unsolved dilemmas: the concept of matter in the bistory of philosophy and in contemporary physics in Information and the nature of reality: From physics to metaphysics, ed. by P. Davies and N. H. Gregersen, Cambridge University Press, 2014.

[2] D. Dürr and S. Teufel, Bobmian mechanics, Springer, 2009.

[3] G. Birkhoff and J. von Neumann, The logic of quantum mechanics, Ann. Math. 37823 (1936).

[4] K. Popper, Conjectures and refutations: The growth of scientific knowledge (Routledge 2014).

[5] G.M. D'Ariano, La Fisica come Informazione: l'universo è un grande automa quantistico, Rendiconti Istituto Lombardo - Accademia di Scienze e Lettere 144 253 (2010).

[6] T. S. Kuhn, The structure of scientific revolutions (University of Chicago press 2012).

[7] G.M. D'Ariano, A Quantum Digital Universe, Il Nuovo Saggiatore 2813 (2012)

[8] G M. D'Ariano, L'Univers est-il un ordinateur quantique?, La Recherche, JuilletAout 2014, n.489, pag. 48.

[9] G.M. D'Ariano, It from Qubit, in A. Aguirre et al. (eds.), It From Bit or Bit From It?, The Frontiers Collection (Springer 2015). 
[10] G.M. D'Ariano, Quantum-Informational Principles for Physics, in A. Aguirre et al. (eds.), Questioning the Foundations of Physics, The Frontiers Collection (Springer 2015).

[11] Feynman lectures on computation, ed. by A. J. G. Hey (Addison-Wesley Longman Publishing Co., Inc. 1998).

[12] J.A. Wheeler, Cosmology, Physics and Philosopby (Springer, NY 1987).

[13] L. Floridi, The philosophy of information (Oxford University Press 2011).

[14] T.W. Bynum, On the possibility of quantum informational structural realism, Minds and Machines 24123 (2014).

[15] B.H. Yandell, The Honors Class-Hilbert?s Problems and Their Solvers, (A. K. Peters, Natick MA 2002).

[16] J.S. Bell, On the Einstein Podolsky Rosen Paradox, Physics 1195 (1964), reproduced as Ch. 2 of J. S. Bell, Speakable and Unspeakable in Quantum Mechanics, (Cambridge University Press 1987).

[17] J.F. Clauser, M.A. Horne, A. Shimony, R.A. Holt, Proposed experiment to test local hidden-variable theories, Phys. Rev. Lett. 23880 (1969).

[18] A. Aspect, P. Grangier, G. Roger, Experimental Tests of Realistic Local Theories via Bell's Theorem, Phys. Rev. Lett. 47460 (1981).

[19] D.B. Malament, In defense of dogma: Why there cannot be a relativistic quantum mechanics of (localizable) particles, in Perspectives on quantum reality (Springer 1996), 1.

[20] H. Halvorson and R. Clifton, No place for particles in relativistic quantum theories?, Philosophy of Science 69 1(2002).

[21] Meinhard Kuhlman, What is real?, Scientific American Aug. 33 (2013).

[22] A. Einstein, B. Podolsky and N. Rosen, Phys. Rev. 47, 777 (1935).

[23] D. Bohm, A Suggested Interpretation of the Quantum Theory in Terms of 'Hidden Variables' I. Phys. Rev. 85166 (1952).

[24] G. Chiribella, G. M. D'Ariano, P. Perinotti, Informational derivation of Quantum Theory, Phys. Rev A 84012311 (2011).

[25] G.M. D'Ariano, G. Chiribella, P. Perinotti, Quantum Theory from Principles, (Cambridge University Press, Cambridge, Boston 2017) (in press).

[26] L. Wittgenstein, Tractatus Logico-Philosophicus. The German Text of LogischPhilosophische Abhandlung, with a new translation by DF Pears \& BF Mcguinness, and with the introduction by Bertrand Russell (Rutledge, London, New York 1974).

[27] E.T. Jaynes, Probability Theory: The Logic of Science (Cambridge University Press, Cambridge 2003).

[28] R.T. Cox, The Algebra of Probable Inference (Johns Hopkins University Press, Baltimore 1961).

[29] N. Herbert, FLASH? a superluminal communicator based upon a new kind of quantum measurement, Found. Phys. 121171 (1982).

[30] D. Kaiser, How the hippies saved physics: science, counterculture, and the quantum revival, (WW Norton \& Company, 2011).

[31] G. Ghirardi, A. Rimini, and T. Weber, Lett. Nuovo Cimento 27293 (1980). 
[32] J. Pearl, Causality, (Cambridge University Press, Cambridge, Boston 2009).

[33] G.M. D'Ariano, Da quali principi discende la Meccanica Quantistica?, Rendiconti Istituto Lombardo - Accademia di Scienze e Lettere 14223 (2008).

[34] G. Chiribella, G. M. D'Ariano, P. Perinotti, Probabilistic Theories with Purification, Phys. Rev. A 81062348 (2010).

[35] G.M. D'Ariano, P. Perinotti, Derivation of the Dirac Equation from Principles of Information processing, Phys. Rev. A 90062106 (2014).

[36] J. Meier, Groups, graphs and trees: an introduction to the geometry of infinite groups, (Cambridge University Press, Cambridge, Boston 2008).

[37] P. de La Harpe, Topics in geometric group theory, (University of Chicago Press, 2000).

[38] J. Stachel, Einstein and the Quantum, in From quarks to quasars: philosophical problems of modern physics, ed. by R. G. Colodny (University of Pittsburgh Press 1986), p. 379.

[39] A. Bisio, G. M. D'Ariano, and P. Perinotti, Quantum cellular automaton theory of light, Ann. Phys. 368177 (2016).

[40] L. De Broglie, Une nouvelle conception de la lumière 181, Hermamm \& Cie (1934). 